\title{
Upper bounds for the ground state energy of the Laplacian with zero magnetic field on planar domains
}

\author{
Bruno Colbois ${ }^{1} \cdot$ Alessandro Savo ${ }^{2}$
}

Received: 15 July 2020 / Accepted: 2 February 2021 / Published online: 15 March 2021

(c) The Author(s) 2021

\begin{abstract}
We obtain upper bounds for the first eigenvalue of the magnetic Laplacian associated to a closed potential 1-form (hence, with zero magnetic field) acting on complex functions of a planar domain $\Omega$, with magnetic Neumann boundary conditions. It is well known that the first eigenvalue is positive whenever the potential admits at least one non-integral flux. By gauge invariance, the lowest eigenvalue is simply zero if the domain is simply connected; then, we obtain an upper bound of the ground state energy depending only on the ratio between the number of holes and the area; modulo a numerical constant the upper bound is sharp and we show that in fact equality is attained (modulo a constant) for AharonovBohm-type operators acting on domains punctured at a maximal $\epsilon$-net. In the last part, we show that the upper bound can be refined, provided that one can transform the given domain in a simply connected one by performing a number of cuts with sufficiently small total length; we thus obtain an upper bound of the lowest eigenvalue by the ratio between the number of holes and the area, multiplied by a Cheeger-type constant, which tends to zero when the domain is metrically close to a simply connected one.
\end{abstract}

Keywords Magnetic Laplacian · Ground state energy · Upper bounds · Planar domains · Zero magnetic field

Mathematics Subject Classification 58J50 · 35P15

Alessandro Savo

alessandro.savo@uniroma1.it

Bruno Colbois

bruno.colbois@unine.ch

1 Université de Neuchâtel, Institut de Mathématiques, Rue Emile Argand 11, CH-2000 Neuchâtel, Suisse

2 Dipartimento SBAI, Sezione di Matematica, Sapienza Università di Roma, Via Antonio Scarpa 16, 00161 Roma, Italy 


\section{Introduction}

We consider a smooth, connected and bounded domain $\Omega \subset \mathbb{R}^{2}$ of area $|\Omega|$. Let $A$ be a closed 1-form and introduce the magnetic Neumann Laplacian $\Delta_{A}$ with potential $A$ acting on functions $u \in C^{\infty}(\Omega, \mathbb{C})$. It is the operator $\Delta_{A}=\left(\nabla^{A}\right)^{\star} \nabla^{A}$ where the connection $\nabla^{A}$ is defined as $\nabla^{A} u=\nabla u-i u A^{\sharp}$ and $A^{\sharp}$ is the vector potential, dual to the 1 -form $A$. The following notation is sometimes used:

$$
\Delta_{A}=\left(i \nabla+A^{\sharp}\right)^{2},
$$

We take (magnetic) Neumann boundary conditions and then we study the eigenvalue problem:

$$
\left\{\begin{array}{l}
\Delta_{A} u=\lambda u \text { on } \Omega \\
\nabla_{N}^{A} u=0 \text { on } \partial \Omega
\end{array}\right.
$$

where $N$ is the inner unit normal. We are interested in the ground state energy (lowest eigenvalue) $\lambda_{1}(\Omega, A)$, which is the minimum of the Rayleigh quotient:

$$
\lambda_{1}(\Omega, A)=\min \left\{\frac{\int_{\Omega}\left|\nabla^{A} u\right|^{2}}{\int_{\Omega}|u|^{2}}: u \in H^{1}(\Omega) \backslash\{0\}\right\} .
$$

If $A=0$, the spectrum of $\Delta_{A}$ coincides with the spectrum of the usual Laplacian under Neumann boundary conditions. The same is true when $A$ is an exact one-form, thanks to the well-known gauge invariance of the magnetic Laplacian, which implies in particular that:

$$
\lambda_{1}(\Omega, A)=\lambda_{1}(\Omega, A+d f)
$$

for any smooth function $f$. The two-form $B \doteq d A$ is called the magnetic field associated to the potential $A$. It turns out that, even if the magnetic field is 0 , the (closed) potential $A$ can affect the ground state energy: this is related to a phenomenon in quantum mechanics called Aharonov-Bohm effect. To see this, we introduce the flux of $A$ across the closed curve (loop) $c$ as the quantity:

$$
\Phi_{c}^{A}=\frac{1}{2 \pi} \oint_{c} A
$$

(we do not specify the orientation of the loop, as it will be irrelevant for our bounds).

It turns out that $\lambda_{1}(\Omega, A)=0$ if and only if $A$ is closed and the cohomology class of $A$ is an integer, that is, the flux of $A$ around any loop is an integer.

This fact was first observed by Shigekawa [10] for closed manifolds and then proved in Theorem 1.1 of [8] for manifolds with boundary. This remarkable feature of the magnetic Neumann Laplacian shows its deep relation with the topology of the underlying domain $\Omega$. For a more detailed introduction to the magnetic Neumann Laplacian associated to a closed potential, see the introduction of [5] and the references therein.

It is precisely the goal of this note to investigate how the topology and the geometry of the domain $\Omega$ influence the ground state energy $\lambda_{1}(\Omega, A)$ when the magnetic field is zero. Therefore, from now on, unless otherwise stated:

- the potential $A$ in this paper will always be a closed one-form. 
Note that, in view of Shigekawa's remark, any lower bound of the ground state energy should somewhat depend on the distance of the fluxes of $A$ to the lattice of integers which, for a single loop $c$, is defined as:

$$
d\left(\Phi_{c}^{A}, \mathbf{Z}\right) \doteq \min \left\{\left|\Phi_{c}^{A}-k\right|, k \in \mathbf{Z}\right\} .
$$

In our previous papers [4] and [5], we obtained lower bounds for the ground state energy. In [4], we proved an estimate from below of the first eigenvalue of a Riemannian cylinder; applied to a plane annulus $\Omega=F \backslash \bar{G}$, with $F$ and $G$ smooth and convex, the lower bound becomes:

$$
\lambda_{1}(\Omega, A) \geq \frac{4 \pi^{2}}{|\partial F|^{2}} \frac{\beta^{2}}{B^{2}} d(\Phi, \mathbf{Z})^{2}
$$

where $\Phi$ is the flux of $A$ across the inner boundary component $\partial G$ and $\beta$ (resp. $B$ ) is the minimal length (resp. the maximal length) of a segment contained in $\Omega$ and hitting the inner boundary $\partial G$ orthogonally. We call $\beta$ and $B$ the minimal, resp. maximal width of $\Omega$, respectively; obviously, $\beta$ is also the minimum distance between the inner and outer boundary curves.

In the subsequent paper [5], we improved the result to get a lower bound depending on $\frac{\beta}{B}$, rather than $\frac{\beta^{2}}{B^{2}}($ see Theorem 1$)$ :

$$
\lambda_{1}(\Omega, A) \geq \frac{\pi^{2}}{8} \frac{|F|^{2}}{|\partial F|^{2} D(F)^{4}} \frac{\beta(\Omega)}{B(\Omega)} \cdot d\left(\Phi^{A}, \mathbf{Z}\right)^{2} .
$$

where $D(F)$ is the diameter of $F$; this linear dependence is in fact sharp, as shown in [5]. We will in fact use (5) in Sect. 5.2, formula (21). In [5], we also extend the lower bound to domains with an arbitrary number of holes.

Upper bounds for the spectrum of the magnetic Schrödinger operator, for an arbitrary potential one-form $A$, were considered in [2]. Some of them are consequence of the inequality $\lambda_{1}(\Omega, A) \leq \mu_{1}(\Omega)$, where $\mu_{1}(\Omega)$ is the lowest eigenvalue of the Schrödinger operator $\Delta+|A|^{2}$, with Neumann boundary conditions. In particular, Theorem 3 in [2] gives an upper bound of the first eigenvalue when the potential is a harmonic one-form, which depends on the volume of $\Omega$ and the distance (taken in $L^{2}$ ) of $A$ to the lattice of integral harmonic one-forms. However, this upper bound is difficult to compute, in general.

The scope of this paper is to prove upper bounds of $\lambda_{1}(\Omega, A)$ which are computable, and depend explicitly on the topology and the geometry of $\Omega$. The topology of a planar domain $\Omega$ is specified by the number $n=n(\Omega)$ of holes, and in fact our first main result, Theorem 1, gives an upper bound of the ground state energy depending only on the area of $\Omega$ and the number of holes; up to a numerical constant, the bound is sharp and is achieved for a certain class of punctured domains (see Theorem 3 ). Note that, if $n=0, \Omega$ is simply connected, $A$ is exact and then $\lambda_{1}(\Omega, A)=0$ : One could intuitively argue that if it is possible to transform a domain $\Omega$ into a simply connected domain by deleting a family of segments of small total length, then $\lambda_{1}(\Omega, A)$ should be small. We somewhat show that in fact this is the case in our second main result, Theorem 6.

We now give the precise statements of our results. 


\section{Main results}

We have already remarked that $\lambda_{k}(\Omega, 0)$ is just the $k$-th eigenvalue of the usual Laplacian with Neumann boundary conditions. Note that, $\lambda_{1}(\Omega, 0)=0$, the associated eigenspace being one-dimensional, spanned by the constant functions. Then, one could ask if $\lambda_{1}(\Omega, A)$ could be somewhat compared with the first positive Neumann eigenvalue, that is, to $\lambda_{2}(\Omega, 0)$ (but in fact we will see that there is no a priori inequality between the two eigenvalues, see below). To that end, recall the Szegö-Weinberger inequality, stating that the Neumann ground state is bounded above by that of the disk $\bar{\Omega}$ of the same area:

$$
\lambda_{2}(\Omega, 0) \leq \lambda_{2}(\bar{\Omega}, 0)
$$

which leads to an upper bound only in terms of the area:

$$
\lambda_{2}(\Omega, 0) \leq \frac{C}{|\Omega|}
$$

where $C=\pi \lambda_{2}(B, 0)$ and $B$ is the unit ball in $\mathbf{R}^{2}$.

Our first question was to see if a weak Szegö-Weinberger inequality could possibly hold for $\lambda_{1}(\Omega, A)$ in this context: that is, can we find an absolute constant $C$ such that for every closed potential $A$ on $\Omega$ one has:

$$
\lambda_{1}(\Omega, A) \leq \frac{C}{|\Omega|} ?
$$

A bit surprisingly, we find out that (7) cannot hold in that generality; the estimate must in fact depend on the topological complexity of $\Omega$, that is, on the number $n=n(\Omega)$ of holes.

Theorem 1 Let $\Omega \subset \mathbb{R}^{2}$ be a bounded domain with smooth boundary having $n$ holes. Then, there exists a universal constant $C>0$ such that for every closed potential $A$, we have:

$$
\lambda_{1}(\Omega, A) \leq C \frac{n}{|\Omega|} .
$$

One could take $C=544 \pi$.

The constant $C$ is not optimal, but modulo a universal constant the upper bound is sharp, as the next result will show. In other words, there are examples of pairs $(\Omega, A)$ with $\Omega$ of fixed area, whose first eigenvalue grows proportionally to the number of holes $n$.

\subsection{Punctured domains and maximal $\epsilon$-nets}

We introduce punctured domains: These are obtained by deleting $n$ given points $\mathcal{P}=\left\{p_{1}, \ldots, p_{n}\right\}$ from a given domain $\Omega$. We define:

$$
\lambda_{1}(\Omega \backslash \mathcal{P}, A)=\liminf _{\eta \rightarrow 0} \lambda_{1}(\Omega \backslash \mathcal{P}(\eta), A)
$$

where $\mathcal{P}(\eta)$ is the $\eta$-neighborhood of $\mathcal{P}$ (it obviously consists of a finite set of closed disks of radius $\eta$ ). It is not our scope in this paper to investigate the convergence in terms of $\eta$. 
A general lower bound for the first eigenvalue of punctured domains is given in Theorem 3 of [5]. An interesting feature of punctured domains, which we will explicit in Sect. 4 and which does not follow trivially from [5], is that their first eigenvalue could grow proportionally to the number of punctures, provided that the configuration $\mathcal{P}$ is a maximal $\epsilon$-net, which we are going to define.

Definition 2 Given a convex domain $\Omega \subset \mathbb{R}^{2}$ with smooth boundary and a number $\epsilon>0$, a maximal collection of points $\mathcal{P}_{\epsilon}=\left\{p_{1}, \ldots, p_{n}\right\}$ with the following properties:

- $d\left(p_{j}, p_{k}\right) \geq \epsilon$ for all $j \neq k$,

- $d\left(p_{j}, \partial \Omega\right) \geq \epsilon$ for all $j$ is called a maximal $\epsilon$-net.

One should think of a maximal $\epsilon$-net as an optimal way of distributing a set of points inside $\Omega$ with the constraint of being at distance at least $\epsilon$ among themselves and at distance at least $\epsilon$ to the boundary. Consider the harmonic 1-form $A$ on $\Omega \backslash \mathcal{P}_{\epsilon}$ which has the same flux $\Phi>0$ around each of the holes $p_{1}, \ldots, p_{n}$. We denote by

$$
d(\Phi, \mathbf{Z})=\min \{|\Phi-k|: k \in \mathbf{Z}\}
$$

the distance of the common flux $\Phi$ to the lattice of integers. We then have:

Theorem 3 If $\partial \Omega$ satisfies the $\delta$-interior ball condition, then, for all $\epsilon<\delta$ and for all maximal $\epsilon$-nets $\mathcal{P}_{\epsilon}$, one has:

$$
\lambda_{1}\left(\Omega \backslash \mathcal{P}_{\epsilon}, A\right) \geq \frac{1}{64} \frac{d(\Phi, \mathbf{Z})^{2}}{\epsilon^{2}}
$$

In terms of the number of points $n=n(\epsilon)$ (hence, the number of holes), we have:

$$
\lambda_{1}\left(\Omega \backslash \mathcal{P}_{\epsilon}, A\right) \geq \frac{\pi}{256} \cdot \frac{n}{|\Omega|} \cdot d(\Phi, \mathbf{Z})^{2} .
$$

The strategy of the proof is to partition the given punctured domain in a family of convex domains with only one puncture and then to apply a lower bound proved in [4] to each piece of the partition.

Recall that $\Omega$ satisfies the $\delta$-interior ball condition if, for any $x \in \partial \Omega$, there exists a ball of radius $\delta$ tangent to $\partial \Omega$ at $x$ and entirely contained in $\Omega$. This is equivalent to saying that the injectivity radius of the normal exponential map is at least $\delta$; hence any point of a segment hitting the boundary orthogonally at $p \in \partial \Omega$ minimizes the distance to the boundary up to distance $\delta$ to $p$.

Remark 4 The referee called our attention to the paper [1] by Balinsky, where a similar estimate is discussed. In fact, Balinsky uses a conformal mapping argument to obtain a lower bound for doubly connected domains in terms of conformal geometry. This bound is similar in the spirit to the lower bound in [4], which we use in our proof here, and which is expressed in terms of explicit geometric quantities.

The referee also pointed out to us the interesting paper [9], where the authors give sharp lower bounds for punctured disks. However, since in our proof, the domains are not punctured disks but punctured convex sets, we have prefered to use our lower bound in [4].

More generally, the inequality holds with $\mathcal{F}^{2}$ replacing $d(\Phi, \mathbf{Z})^{2}$, where 


$$
\mathcal{F}^{2}=\min _{j=1, \ldots, n} d\left(\Phi_{j}, \mathbf{Z}\right)^{2}
$$

and $\Phi_{j}$ is the flux of $A$ around $p_{j}$.

Assuming constant flux $\frac{1}{2}$ around every point of the net, we see that the domain $\Omega \backslash \mathcal{P}_{\epsilon}$ (having $n$ holes and area $|\Omega|$ ) satisfies the bounds:

$$
\frac{1}{1024} \leq \lambda_{1}\left(\Omega \backslash \mathcal{P}_{\epsilon}, A\right) \cdot \frac{|\Omega|}{n} \leq 544,
$$

showing that (8), modulo a numerical constant, is sharp.

A final question in this regard is the following:

- Is there an inequality relating $\lambda_{1}(\Omega, A)$ with $\lambda_{2}(\Omega, 0)$ ?

The answer is negative. To show this, first consider that when $\epsilon$ is sufficiently small and $0<\eta<\frac{\epsilon}{2}$, one has, in the previous notation:

$$
\lambda_{1}\left(\Omega \backslash \mathcal{P}_{\epsilon}(\eta), A\right)>\lambda_{2}\left(\Omega \backslash \mathcal{P}_{\epsilon}(\eta), 0\right)
$$

where $\mathcal{P}_{\epsilon}(\eta)$ is the $\eta$-neighborhood of $\mathcal{P}_{\epsilon}$. In fact, as $\epsilon \rightarrow 0$, the left-hand side diverges to infinity while the right-hand side is uniformly bounded above by the Szegö-Weinberger inequality (6).

In the other direction, remove from a fixed rectangle $F$ in the plane another smaller rectangle $G$ with fixed sides parallel to those of $F$, such that the boundary components of $F$ and $G$ get $\epsilon$-close to each other: see Fig. 2 in [5]. There it is proved that the resulting domain $\Omega_{\epsilon}$ is such that $\lambda_{1}\left(\Omega_{\epsilon}, A\right)$ converges to zero proportionally to $\epsilon$, where $A$ is the closed potential having flux $\frac{1}{2}$ around the inner curve $\partial G$. Nevertheless, one observes that the Cheeger constant of $\Omega_{\epsilon}$ is uniformly bounded below by a positive constant $C$, which implies that $\lambda_{2}\left(\Omega_{\epsilon}, 0\right) \geq C>0$. Therefore, for $\epsilon$, small one has $\lambda_{1}\left(\Omega_{\epsilon}, A\right)<\lambda_{2}(\Omega, 0)$.

We remark that in [6], the authors investigate the validity of the Szegö-Weinberger inequality when the magnetic potential is nonzero (in particular, has constant norm).

\subsection{An upper bound by a Cheeger type constant}

First observe that, if $\Omega$ has $n$ holes, one can suitably delete $n$ segments from $\Omega$ (joining different connected components of the boundary) and get a simply connected domain. We will establish an upper bound of $\lambda_{1}$ depending on the sum of the lengths of these segments, denoted by $h(\Omega)$. On one side, it will show that if $h(\Omega)$ is small enough, then the upper bound we get is better than the bound of Theorem 1; on the other hand, we will construct an example showing that even if $h(\Omega)$ goes to $0, \lambda_{1}(\Omega, A)|\Omega|$ may be large (and therefore the number of holes must be large).

Definition 5 Let $\Omega \subset \mathbb{R}^{2}$ be a bounded domain. An admissible cut of $\Omega$ is a collection of segments $\Gamma=\left\{\Gamma_{1}, \ldots, \Gamma_{n}\right\}$ such that $\Omega \backslash \Gamma$ is simply connected. Introduce the constant $h(\Omega)$ :

$$
h(\Omega)=\min \left\{\sum_{i=1}^{n} h_{i}: h_{i}=\operatorname{length}\left(\Gamma_{i}\right)\right\}
$$

where $\Gamma$ is a admissible cut of $\Omega$. 
The constant $h$ may be seen as an adapted Cheeger constant to measure how the topology (the number $n=n(\Omega)$ of holes) and the geometry (the lengths $h_{j}$ of the segments $\Gamma_{j}$ ) interact in order to affect the first eigenvalue $\lambda_{1}(\Omega, A)$. A natural question is for example to ask how small $h$ must be in comparison with $n(\Omega)$ in order to guarantee that $\lambda_{1}(\Omega, A)$ is uniformly bounded for a family $\Omega$ of domains of given area with a fixed number of holes.

Theorem 6 Assume that $h(\Omega) \leq \frac{|\Omega|}{2 \pi}$ and $h_{j} \leq 1$ for each $j=1, \ldots n$. Then:

$$
\lambda_{1}(\Omega, A) \leq \frac{8 \pi n(\Omega)}{|\Omega|} \sum_{j=1}^{n} \frac{1}{\left|\ln \frac{h_{j}}{2}\right|}
$$

where $h_{j}$ denotes the length of the $j$-th segment $\Gamma_{j}$ associated to $h$ and $n(\Omega)$ is the number of holes.

Note that, we assume bounds on $\frac{h}{|\Omega|}$ and on every $h_{j}$ : This is a technical fact needed for the proof. On the other hand, Theorem 6 is meaningful and improves Theorem 1 in the special situation where $h_{j}$ and $h$ are very small; the general situation is treated in Theorem 1 , which does not follow from Theorem 6.

Corollary 7 In particular, assume that $\Omega$ is doubly connected. If $h \leq \min \left\{1, \frac{|\Omega|}{2 \pi}\right\}$, then

$$
\lambda_{1}(\Omega, A) \leq \frac{8 \pi}{|\Omega|\left|\ln \frac{h}{2}\right|}
$$

Note that, for doubly connected domains, one has $h=\beta$ where $\beta$ is the minimal width of $\Omega$, and also the minimum distance between the two boundary components. The corollary shows that if $|\Omega|$ is fixed and the boundary components get very close (that is, $h=\beta$ tends to zero), then $\lambda_{1}$ tends to zero, which indeed improves Theorem 1. An interesting question is to see if the rate at which this happens, that is $1 /\left|\ln \frac{h}{2}\right|$, is actually sharp or can be improved.

When there is more than one hole, it is still possible to have an upper bound directly in terms of $h(\Omega)$.

Corollary 8 Assume in addition that, in the definition of $h$, every $h_{j} \leq e^{-2}$. Then, we have:

$$
\lambda_{1}(\Omega, A) \leq \frac{8 \pi n(\Omega)^{2}}{|\Omega|} \frac{1}{\left|\ln \left(\frac{h(\Omega))}{n(\Omega)}\right)\right|} .
$$

For example, if $\Omega$ has area 1 and $n$ holes, in order to guarantee that $\lambda_{1}(\Omega, A) \leq 1$, we need to impose $h(\Omega) \leq n e^{-8 \pi n^{2}}$.

It is natural to ask what occurs when $h \rightarrow 0$ for domains of given area. Clearly, if $n(\Omega)$ is fixed and $h \rightarrow 0$, inequality (12) implies that $\lambda_{1}(\Omega, A) \rightarrow 0$.

However, if $n(\Omega)$ is not fixed, the assumption $h \rightarrow 0$ does not imply that the first eigenvalue tends to zero. In fact, we can have $h$ arbitrarily small and, at the same time, $\lambda_{1}(\Omega, A)|\Omega|$ as large as one wishes. The next example is an illustration of this fact.

Example 9 There exists a family of domains $\left\{\Omega_{k}\right\}_{k \geq 1}$ with area $\left|\Omega_{k}\right| \geq 1$ and with a fixed potential $A$ of equal flux $\Phi>0$ around each hole such that 


$$
h\left(\Omega_{k}\right) \leq \frac{2}{\sqrt{k}}
$$

and, at the same time:

$$
\lambda_{1}\left(\Omega_{k}, A\right) \geq c \sqrt{k} d(\Phi, \mathbf{Z})^{2}
$$

with $c=\frac{\pi^{2}}{2^{15} \sqrt{2}}$. The number of holes of $\Omega_{k}$ is $n\left(\Omega_{k}\right)=k^{2}$ (hence, it grows with $k$ ).

\section{Proof of Theorem 1}

Recall that we want to show that if $\Omega \subset \mathbb{R}^{2}$ is a bounded domain with smooth boundary having $n$ holes then, for every closed potential $A$, we have:

$$
\lambda_{1}(\Omega, A) \leq 544 \pi \frac{n}{|\Omega|} .
$$

Proof The proof consists in three steps. First, using gauge invariance, we replace the given potential $A$ by a new potential having the same flux but with poles at a certain collection of points $\left\{p_{1}, \ldots, p_{n}\right\}$. The two corresponding magnetic Laplacians are unitarily equivalent and have the same spectrum. In the second step, we show the existence of a ball $B(p, r)$ of radius $r=\frac{1}{4 \sqrt{\pi}}\left(\frac{|\Omega|}{n}\right)^{\frac{1}{2}}$ such that for each $i=1, \ldots, n, p_{i} \notin B(p, 2 r)$. Moreover, we get a control of the area growth by the relation

$$
\frac{|B(p, 2 r) \cap \Omega|}{|B(p, r) \cap \Omega|} \leq 34
$$

In the last step, the fact that $p_{i} \notin B(p, 2 r)$ for any $i$ will imply that $A$ is exact on $B(p, 2 r)$, hence, thanks to the control of the volume growth of the balls, one can control $\lambda_{1}(\Omega, A)$ by a standard cutoff argument for the usual Laplacian.

Step 1. The domain $\Omega$ is bounded by an outer closed curve $\Sigma_{0}$ and $n$ closed inner curves $\Sigma_{1}, \ldots, \Sigma_{n}$. We assume that our closed potential $A$ has flux $\Phi_{i}^{A}$ around $\Sigma_{i}$.

We choose $n$ points $p_{1}, \ldots, p_{n}$ so that $p_{i}$ is inside $\Sigma_{i}$, and we write $\left(a_{i}, b_{i}\right)$ for the coordinates of $p_{i}$. Let $A_{i}$ be the 1 -form

$$
A_{i}(x, y)=\Phi_{i}^{A}\left(\frac{-\left(y-b_{i}\right)}{\left(x-a_{i}\right)^{2}+\left(y-b_{i}\right)^{2}} d x+\frac{\left(x-a_{i}\right)}{\left(x-a_{i}\right)^{2}+\left(y-b_{i}\right)^{2}} d y\right) .
$$

The flux of $A_{i}$ is equal to $\Phi_{i}^{A}$ around $\Sigma_{i}$ and it is 0 around $\Sigma_{j}$ for $j \neq i$ (we assume that every $\Sigma_{i}$ is traveled once). This implies that the fluxes associated to the 1 -form $\tilde{A} \doteq A_{1}+\ldots+A_{n}$ are equal to the fluxes of $A$, and therefore $A-\tilde{A}$ is exact. By Gauge invariance, the operators $\Delta_{A}$ and $\Delta_{\tilde{A}}$ have the same spectrum, so that it suffices to find an upper bound for $\lambda_{1}(\Omega, \tilde{A})$.

Step 2. First, we prove the following estimate that will be used in the proof of Lemma 10 below. Let $a>b$ and consider the maximal number $N=N(a, b)$ of points at distance at least $b$ from one another which are in a ball $B(a)$ of radius $a$. We find: 


$$
\left(\frac{a}{b}\right)^{2} \leq N \leq\left(\frac{2 a+b}{b}\right)^{2}
$$

To see this estimate, we denote by $x_{1}, \ldots, x_{N}$ a maximal net of points at mutual distance at least $b$ in the ball $B(a)$. The balls of center $x_{i}$ and radius $\frac{b}{2}$ are disjoint and contained in the ball of radius $a+\frac{b}{2}$, so that

$$
\sum_{i=1}^{N}\left|B\left(x_{i}, \frac{b}{2}\right)\right| \leq\left|B\left(a+\frac{b}{2}\right)\right|
$$

which means that $N(a, b) \frac{b^{2}}{4} \pi \leq \pi\left(\frac{2 a+b}{2}\right)^{2}$, and then:

$$
N(a, b) \leq\left(\frac{2 a+b}{b}\right)^{2} .
$$

On the other hand, by maximality, the union of the balls $B\left(x_{i}, b\right)$ covers $B(a)$, and

$$
|B(a)| \leq \sum_{i=1}^{N}\left|B\left(x_{i}, b\right)\right|
$$

so that $\pi a^{2} \leq N(a, b) \pi b^{2}$ and $N(a, b) \geq \frac{a^{2}}{b^{2}}$ as asserted. This proves (14).

In order to construct an upper bound for $\lambda_{1}(\Omega, \tilde{A})$, we will construct a test function with Rayleigh quotient $\leq C \frac{n}{|\Omega|}$. This test function will be constructed geometrically with ideas coming from [3], but much easier to apply in our case, because we are concerned only with the first eigenvalue. Fix the number:

$$
r \doteq \frac{1}{4 \sqrt{\pi}}\left(\frac{|\Omega|}{n}\right)^{\frac{1}{2}} .
$$

Then, we have the following fact.

Lemma 10 There exists a point $p \in \Omega$ such that $p_{j} \notin B(p, 2 r)$ for every $j=1, \ldots, n$, and moreover

$$
\frac{|B(p, 2 r) \cap \Omega|}{|B(p, r) \cap \Omega|} \leq 34 .
$$

Proof of the lemma From the definition of $r$ one sees that

$$
\sum_{j=1}^{n}\left|B\left(p_{j}, 2 r\right)\right| \leq n \pi(2 r)^{2} \leq 4 \pi n \frac{1}{16 \pi}|\Omega| \frac{1}{n}=\frac{|\Omega|}{4} .
$$

Set

$$
\Omega_{0}=\Omega \backslash \cup_{j} B\left(p_{j}, 2 r\right),
$$

so that

$$
\left|\Omega_{0}\right| \geq \frac{3}{4}|\Omega|
$$


For any $q \in \Omega_{0}$, we have clearly $p_{j} \notin B(q, 2 r)$ for all $j$. Take a maximal $r$-net in $\Omega_{0}$, say $\mathcal{N}=\left\{q_{1}, \ldots, q_{m}\right\}$, so that $d\left(q_{i}, q_{j}\right) \geq r$ for all $i, j$ and by the maximality of the net:

$$
\Omega_{0} \subset \cup_{j=0}^{m} B\left(q_{j}, r\right) .
$$

This implies that

$$
\left.\sum_{j=1}^{m} \mid B\left(q_{j}, r\right) \cap \Omega\right)|\geq| \Omega_{0}\left|\geq \frac{3}{4}\right| \Omega \mid .
$$

By the estimate (14), for any $q \in \Omega$, the cardinality of the set $\mathcal{N} \cap B(q, 2 r)$ is at most $\left(\frac{2 a+b}{b}\right)^{2}$, with $a=2 r$ and $b=r$, that is:

$$
|\mathcal{N} \cap B(q, 2 r)| \leq 25 \text {. }
$$

In other words, every point $q \in \Omega$ is in at most 25 balls of radius $2 r$ centered at a point of the net, hence

$$
\sum_{j=0}^{m}\left|B\left(q_{j}, 2 r\right) \cap \Omega\right| \leq 25|\Omega| .
$$

We can now prove that there exist $q_{j}$ such that

$$
\frac{\left|B\left(q_{j}, 2 r\right) \cap \Omega\right|}{\left|B\left(q_{j}, r\right) \cap \Omega\right|} \leq 34 .
$$

Assume not. Then:

$$
\left|B\left(q_{j}, 2 r\right) \cap \Omega\right|>34\left|B\left(q_{j}, r\right) \cap \Omega\right|
$$

for all $j$. We would then have, by (15), (16) and (17):

$$
\begin{aligned}
25|\Omega| & \geq \sum_{j=0}^{m}\left|B\left(q_{j}, 2 r\right) \cap \Omega\right| \\
& >34 \sum_{j=1}^{m}\left|B\left(q_{j}, r\right) \cap \Omega\right| \\
& \geq 34 \cdot \frac{3}{4}|\Omega| \\
& >25|\Omega| .
\end{aligned}
$$

which is a contradiction. The lemma is then proved.

Step 3. We take a ball $B(p, 2 r)$ as in Lemma 10. Then, we can conclude as follows. First, the restriction of $\tilde{A}=A_{1}+\ldots+A_{n}$ to $B(p, 2 r)$ is exact, because the poles $p_{1}, \ldots, p_{n}$ are not contained in the ball. Up to a Gauge transformation, we can replace the magnetic Laplacian $\Delta_{\tilde{A}}$ by the usual Laplacian on $B(p, 2 r)$.

We define a function $u: \Omega \rightarrow \mathbf{R}$ as follows: 


$$
u(x)=\left\{\begin{array}{l}
1 \quad \text { if } \quad d(p, x) \leq r \\
-\frac{1}{r} d(p, x)+2 \quad \text { if } \quad d(p, x) \geq r
\end{array}\right.
$$

Note that, $u$ is indeed supported on $B(p, 2 r)$; extending it to zero on the complement of the ball, we get a well-defined test function. As $|\nabla u| \leq \frac{1}{r}$, we see:

$$
\int_{\Omega}|\nabla u|^{2} \leq \frac{1}{r^{2}}|B(p, 2 r) \cap \Omega| .
$$

On the other hand:

$$
\int_{\Omega}|u|^{2} \geq|B(p, r) \cap \Omega|
$$

Hence, its Rayleigh quotient is bounded above as follows:

$$
R(u) \leq \frac{1}{r^{2}} \frac{|B(p, 2 r) \cap \Omega|}{|B(p, r) \cap \Omega|} \leq \frac{34}{r^{2}} .
$$

Recalling the definition of $r$, we conclude:

$$
R(u) \leq 544 \pi \frac{n}{|\Omega|}
$$

as asserted.

\section{Proof of Theorem 3}

The strategy of the proof is to partition the given punctured domain in a family of convex domains with only one puncture and then to apply the lower bound (4) to each piece of the partition.

First, we say that the family of open sets $\left\{\Omega_{1}, \ldots, \Omega_{n}\right\}$ with piecewise-smooth boundary is a partition of the open set $\Omega$ if $\bar{\Omega}=\cup_{j=1}^{n} \bar{\Omega}_{j}$; the partition is disjoint if moreover $\Omega_{j} \cap \Omega_{k}$ is empty whenever $j \neq k$. It is a simple consequence of the min-max principle that the first eigenvalue of $\Omega$ is controlled from below by the smallest first eigenvalue of the members of a disjoint partition, that is:

$$
\lambda_{1}(\Omega, A) \geq \min _{j=1, \ldots, n} \lambda_{1}\left(\Omega_{j}, A\right)
$$

for any potential one-form $A$ (for the easy proof we refer to Proposition 4 of [5]). The second ingredient is the estimate (4) for an annulus $\Omega=F \backslash \bar{G}$ with $F$ and $G$ convex with piecewise-smooth boundary:

$$
\lambda_{1}(\Omega, A) \geq \frac{4 \pi^{2}}{|\partial F|^{2}} \frac{\beta^{2}}{B^{2}} d(\Phi, \mathbf{Z})^{2}
$$

where $\Phi$ is the flux of $A$ across the inner boundary component $\partial G$ and $\beta$ (resp. $B$ ) is the minimal and maximal width of $\Omega$, respectively. 
Let us then start from the partition. In fact, the properties of a maximal $\epsilon$-net allow to partition the given domain in "well-balanced" convex pieces, in the following sense.

Lemma 11 Let $\Omega$ be a convex domain with smooth boundary and let $\mathcal{P}_{\epsilon}=\left\{p_{1}, \ldots, p_{n}\right\}$ be a maximal $\epsilon$-net in $\Omega$. We assume that $\partial \Omega$ satisfies the $\delta$-interior ball condition with $\delta>\epsilon$. Then, $\Omega$ admits a disjoint partition $\left\{\Omega_{1}, \ldots, \Omega_{n}\right\}$ with the following properties:

a) Every $\Omega_{j}$ is convex and has piecewise-smooth boundary;

b) For each $j=1, \ldots$, one has $B\left(p_{j}, \frac{\epsilon}{2}\right) \subseteq \Omega_{j} \subseteq B\left(p_{j}, 2 \epsilon\right)$.

We will prove the lemma below.

To finish the proof of Theorem 3, we first observe that $\left\{\Omega_{1} \backslash\left\{p_{1}\right\}, \ldots, \Omega_{n} \backslash\left\{p_{n}\right\}\right\}$ is a disjoint partition of the punctured domain $\Omega \backslash \mathcal{P}_{\epsilon}$ and, in view of (18), it is enough to bound from below the ground state energy of every piece of this partition. To that end, we apply (19) to $\Omega_{j} \backslash\left\{p_{j}\right\}$, more precisely, we take $F=\Omega_{j}, G=B\left(p_{j}, \eta\right)$ and let $\eta \rightarrow 0$. Taking into account Lemma 11, we have, after taking the limit as $\eta \rightarrow 0$ :

$$
\beta \geq \frac{\epsilon}{2}, \quad B \leq 2 \epsilon, \quad \text { hence } \quad \frac{\beta}{B} \geq \frac{1}{4}
$$

because $\beta$ and $B$ tend, respectively, to the minimum and maximum distance of $p_{j}$ to $\partial \Omega_{j}$. Moreover, as $\Omega_{j}$ is convex, contained in $B\left(p_{j}, 2 \epsilon\right)$, we have by the monotonicity of the perimeter: $\left|\partial \Omega_{j}\right| \leq 4 \pi \epsilon$. The conclusion is that, for all $j=1, \ldots, n$ :

$$
\lambda_{1}\left(\Omega_{j} \backslash\left\{p_{j}\right\}, A\right) \geq \frac{1}{64 \epsilon^{2}} d(\Phi, \mathbf{Z})^{2} .
$$

As this holds for any member of the partition, it holds a fortiori for $\Omega \backslash \mathcal{P}_{\epsilon}$, which proves the first part of the theorem.

Finally, it is readily seen that the number of points in a maximal $\epsilon$-net grows proportionally to $\epsilon^{-2}$. Precisely, one first observes that $\cup_{j=1}^{n} B\left(p_{j}, \frac{\epsilon}{22}\right) \subseteq \Omega$; since the union on the left is disjoint (by maximality of the net) we obtain: $n \cdot \frac{\pi \epsilon^{22}}{4} \leq|\Omega|$, that is

$$
\frac{1}{\epsilon^{2}} \geq \frac{\pi n}{4|\Omega|}
$$

which proves (9).

\subsection{Proof of Lemma 11}

We will use the following property of maximal $\epsilon$-nets:

Property P. If $x \in \Omega$ is such that $d\left(x, p_{j}\right)>\epsilon$ and $d(x, \partial \Omega) \geq \epsilon$, then there exists $p_{k} \neq p_{j}$ such that $d\left(x, p_{k}\right) \leq \epsilon$.

For each $j=1, \ldots, n$ we consider the non-empty open set:

$$
\Omega_{j}=\left\{x \in \Omega: d\left(x, p_{j}\right)<d\left(x, p_{k}\right) \text { for all } k \neq j\right\} .
$$

It is clear that $\bar{\Omega}=\cup_{j=1}^{n} \bar{\Omega}_{j}$. If, for indices $j \neq k$, we consider the open half-space 


$$
H_{j k}=\left\{x \in \mathbf{R}^{2}: d\left(x, p_{j}\right)<d\left(x, p_{k}\right)\right\}
$$

we see that we can write

$$
\Omega_{j}=\cap_{k \neq j}\left(H_{j k} \cap \Omega\right)
$$

which makes it clear that $\Omega_{j}$ is convex. As the boundary of $\Omega_{j}$ is either part of $\partial \Omega$, or is part of $\partial H_{j k}$, which is a straight line, we see that $\partial \Omega_{j}$ is piecewise-smooth. This proves a). We now prove the first inclusion in b). Assume $d\left(x, p_{j}\right)<\frac{\epsilon}{2}$ : it is enough to show that $x \in \bar{\Omega}_{j}$. In fact, if $x \notin \bar{\Omega}_{j}$, there exists $k \neq j$ such that $x \in \bar{\Omega}_{k}$ and, by definition, $d\left(x, p_{k}\right) \leq d\left(x, p_{j}\right)<\frac{\epsilon}{2}$. This means that

$$
d\left(x, p_{j}\right)<\frac{\epsilon}{2} \quad \text { and } \quad d\left(x, p_{k}\right)<\frac{\epsilon}{2}
$$

which by the triangle inequality gives $d\left(p_{j}, p_{k}\right)<\epsilon$, which is a contradiction. Hence, $x \in \bar{\Omega}_{j}$.

We now prove the second inclusion in b). Let $x \in \Omega_{j}$. It is enough to show that $d\left(x, p_{j}\right)<2 \epsilon$ in any of the following two cases:

Case I: $d(x, \partial \Omega) \geq \epsilon$,

Case II: $d(x, \partial \Omega)<\epsilon$.

In Case I, assume that $d\left(x, p_{j}\right) \geq 2 \epsilon$, so that, in particular, $d\left(x, p_{j}\right)>\epsilon$. By Property $\mathrm{P}$ above, there exists $p_{k} \neq p_{j}$ such that $d\left(x, p_{k}\right) \leq \epsilon$. As $x \in \Omega_{j}$ we have, by definition, $d\left(x, p_{j}\right)<d\left(x, p_{k}\right)$ hence a fortiori $d\left(x, p_{j}\right)<\epsilon$ which is a contradiction.

Now assume we are in Case II. Let $\bar{x} \in \partial \Omega$ be the foot of the unique geodesic segment $\gamma$ which minimizes distance to the boundary, and let $p$ be a point of $\gamma$ at distance $\epsilon$ to $\bar{x}$. Since $\partial \Omega$ has the $\delta$-interior ball condition, and since $\epsilon<\delta$, it is clear that

$$
d(p, \partial \Omega)=\epsilon \quad \text { and } \quad d(p, x) \leq \epsilon .
$$

Since in particular $d(p, \partial \Omega) \geq \epsilon$, there exists $p_{k} \in \mathcal{P}_{\epsilon}$ such that $d\left(p, p_{k}\right) \leq \epsilon$, by the $\epsilon$-maximality of the net. By the triangle inequality:

$$
d\left(x, p_{k}\right) \leq d(x, p)+d\left(p, p_{k}\right) \leq 2 \epsilon .
$$

On the other hand, $x \in \Omega_{j}$ and because of that one has $d\left(x, p_{j}\right)<d\left(x, p_{k}\right)$. Hence, $d\left(x, p_{j}\right)<2 \epsilon$ and the proof is complete.

\section{Bound of $\lambda_{1}(\Omega, A)$ with respect to the invariant $h(\Omega)$}

The goal of this section is to prove Theorem 6 and to construct Example 9. This example show that, surprisingly, when the number of holes increase, the constant $h$ can decrease to 0 , and, at the same time, the ground state energy can increase to $\infty$.

\subsection{Proof of Theorem 6.}

Proof First, we observe that, by hypothesis, we can cut $n$ segments $\Gamma_{1}, \ldots, \Gamma_{n}$ in $\Omega$ so that the complement $\Omega \backslash\left\{\Gamma:=\Gamma_{1} \cup \ldots \cup \Gamma_{n}\right\}$ is simply connected.

Let $\Gamma(\epsilon)$ be the $\epsilon$-neighborhood of $\Gamma$ and set $D=\Omega \backslash \Gamma(\epsilon)$; if $\epsilon$ is small enough $D$ is simply connected and we have, by Proposition 12 in [4]: 


$$
\lambda_{1}(\Omega, A) \leq v_{1}(D)
$$

where $v_{1}(D)$ denotes the first eigenvalue of a mixed problem on $D$, where we take the Dirichlet condition on $\partial D \cap \Omega$ and the Neumann condition on $\partial D \cap \partial \Omega$.

In order to control $v_{1}(D)$, we will construct a test function $u$ taking the value 0 on $\partial D \cap \Omega$ and apply the min-max principle.

We assume $\pi h=\pi \sum_{i=1}^{n} h_{i} \leq \frac{|\Omega|}{2}$ and $h_{i} \leq 1$ (Fig. 1).

We consider one of the segments $\Gamma_{i}$ of length $h_{i}$ and denote by $q_{i}$ the middle of $\Gamma_{i}$.

Observe that for $\epsilon$ small enough, $\Gamma(\epsilon) \subset B\left(q_{1}, h_{1}\right) \cup \ldots \cup B\left(q_{n}, h_{n}\right)$. We will construct a test-function $u$ taking the value 0 on $B\left(q_{1}, h_{1}\right) \cup \ldots \cup B\left(q_{n}, h_{n}\right)$, so that it takes the value 0 on $\partial D \cap \Omega$.

We introduce the radial function $u_{i}$ on $D$ defined by

$$
u_{i}(x)=\left\{\begin{array}{l}
0 \quad \text { if } \quad d\left(x, q_{i}\right) \leq h_{i}, \\
\frac{-2}{\ln h_{i}}\left(\ln d\left(x, q_{i}\right)-\ln h_{i}\right) \quad \text { if } \quad h_{i} \leq d\left(x, q_{i}\right) \leq \sqrt{h_{i}}, \\
1 \quad \text { if } \quad d\left(x, q_{i}\right) \geq \sqrt{h_{i}} .
\end{array}\right.
$$

Our test function $u$ on $D$ will be the product:

$$
u(x)=u_{1}(x) \cdots u_{n}(x) .
$$

Taking into account that $\left|u_{i}(x)\right| \leq 1$, we have

$$
|\nabla u| \leq\left|\nabla u_{1}\right|+\ldots+\left|\nabla u_{n}\right|
$$

and therefore

$$
|\nabla u|^{2} \leq n\left(\left|\nabla u_{1}\right|^{2}+\ldots+\left|\nabla u_{n}\right|^{2}\right) .
$$

So it suffices to bound from above the contribution of each $\int_{D}\left|\nabla u_{i}\right|^{2}$ in order to control $\int_{D}|\nabla u|^{2}$. In polar coordinates centered at $q_{i}$, one has:

$$
\left|\nabla u_{i}\right|^{2}=\frac{4}{\ln ^{2} h_{i}} \cdot \frac{1}{r^{2}}
$$

on the subset where $h_{i} \leq r \leq \sqrt{h_{i}}$, and zero everywhere else. Then:
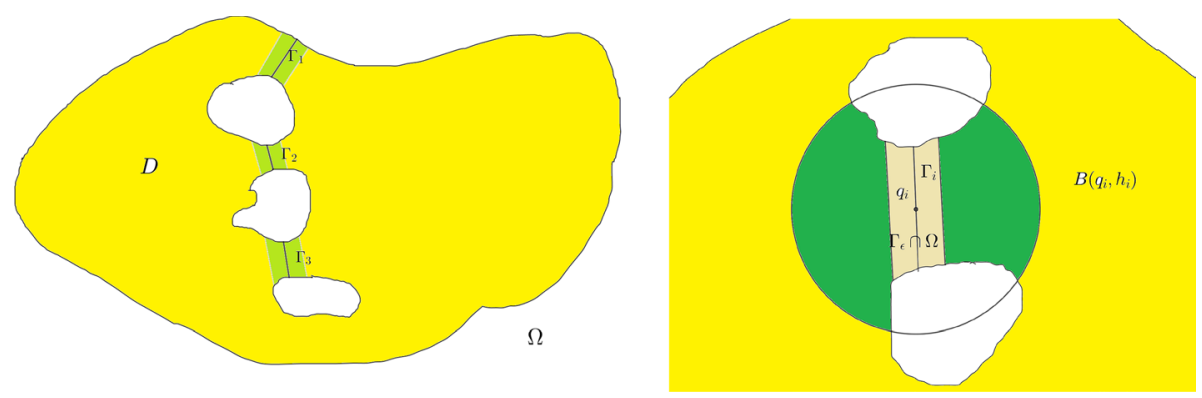

Fig. 1 On the left the domain $D=\Omega \backslash \Gamma_{\epsilon}$. On the right, the ball $B\left(q_{i}, h_{i}\right)$ and its intersection with $\Gamma_{\epsilon}$ 


$$
\int_{D}\left|\nabla u_{i}\right|^{2} \leq 2 \pi \frac{4}{\ln ^{2} h_{i}} \int_{h_{i}}^{\sqrt{h_{i}}} \frac{d r}{r}=\frac{8 \pi}{\ln ^{2} h_{i}}\left(\ln \sqrt{h_{i}}-\ln h_{i}\right)=\frac{-4 \pi}{\ln h_{i}}
$$

hence summing over $i$ (recall that $\left.h_{i} \leq 1\right)$ :

$$
\int_{D}|\nabla u|^{2} \leq 4 \pi n \sum_{i=1}^{n} \frac{-1}{\ln h_{i} / 2}=4 \pi n \sum_{i=1}^{n} \frac{1}{\left|\ln h_{i} / 2\right|}
$$

Taking into account that the area of a ball of radius $\sqrt{h_{i}}$ is $\pi h_{i}$ and that $u=1$ outside these balls, the $L^{2}$-norm of the function $u$ is at least

$$
\int_{\Omega} u^{2} \geq|\Omega|-\pi \sum_{i=1}^{n} h_{i} \geq \frac{|\Omega|}{2}
$$

because we assume $\sum_{i} h_{i}=h \leq \frac{|\Omega|}{2 \pi}$. So, by the min-max principle, we deduce

$$
\lambda_{1}(\Omega, A) \leq \frac{8 \pi n}{|\Omega|} \sum_{i=1}^{n} \frac{1}{\left|\ln h_{i} / 2\right|}
$$

as asserted.

Proof of Corollary 8 First observe that

$$
\frac{1}{\ln \frac{2}{h_{j}}} \leq-\frac{1}{\ln h_{j}}=\frac{1}{\left|\ln h_{j}\right|}
$$

Then, since the function $\phi(x)=-\frac{1}{\ln x}$ is concave $\left(\phi^{\prime \prime}(x) \leq 0\right)$ on the interval $\left(0, e^{-2}\right)$, we have, by Jensen inequality:

$$
\sum_{j=1}^{n} \phi\left(h_{j}\right) \leq n \phi\left(\frac{1}{n} \sum_{j=1}^{n} h_{j}\right)
$$

Translated to our situation, we see that if every $h_{j}<e^{-2}$, then:

$$
\sum_{j=1}^{n} \frac{1}{\left|\ln \frac{2}{h_{j}}\right|} \leq \frac{n}{\left|\ln \left(\frac{h(\Omega)}{n}\right)\right|}
$$

and the upper bound (10) reads:

$$
\lambda_{1}(\Omega, A) \leq \frac{8 \pi n^{2}}{|\Omega|} \frac{1}{\left|\ln \left(\frac{h(\Omega)}{n}\right)\right|} .
$$

where $h(\Omega)$ is the Cheeger constant introduced in Definition 5.

\subsection{Construction of an example}

We will construct a family of domains $\Omega_{k}$ with $n\left(\Omega_{k}\right)=k^{2}$ holes; each $\Omega_{k}$ is obtained as a union of $k^{2}$ identical fundamental pieces $C_{k}$. Each fundamental piece $C_{k}$ is a doubly convex 
domain, so that we will be able to use the inequality of Theorem 1 in [5] to bound from below its first eigenvalue.

Step 1: the definition of the fundamental piece $C_{k}$.

The domain $C_{k}$ is determined by the exterior boundary curve, a square of sidelength $\frac{4}{k}$ based on the vertices

$$
A=\left(-\frac{2}{k}, 0\right), \quad B=\left(\frac{2}{k}, 0\right), \quad C=\left(\frac{2}{k}, \frac{4}{k}\right), \quad D=\left(-\frac{2}{k}, \frac{4}{k}\right),
$$

and the inner boundary curve, a rectangle based on the vertices

$$
A^{\prime}=\left(-\frac{1}{k}, \frac{1}{k^{5 / 2}}\right), \quad B^{\prime}=\left(\frac{1}{k}, \frac{1}{k^{5 / 2}}\right), \quad C^{\prime}=\left(\frac{1}{k}, \frac{4}{k}-\frac{1}{k^{5 / 2}}\right), \quad D^{\prime}=\left(-\frac{1}{k}, \frac{4}{k}-\frac{1}{k^{5 / 2}}\right) .
$$

We refer to the picture below (Fig. 2).

We have:

- the area of $C_{k}$ is $\left|C_{k}\right|=\frac{8}{k^{2}}+\frac{4}{k^{7 / 2}}$;

- the area of the domain $F_{k}$ bounded by the outer curve is $\frac{16}{k^{2}}$;

- the minimal width $\beta_{k}$ between the two boundaries is $\beta_{k}=\frac{1}{k^{5 / 2}}$;

- the maximal width $B_{k}$ between the two boundaries is $B_{k}=\frac{1}{k} \sqrt{1+\frac{1}{k^{3}}}$;

- the diameter of $F_{k}$ is $D_{k}=\frac{4 \sqrt{2}}{k}$;

- the length $L_{k}$ of the exterior boundary is $L_{k}=\frac{16}{k}$.

We consider a potential $A$ with flux $\Phi$. We can apply again Theorem 1 in [5] (see (5)) and we obtain:

$$
\lambda_{1}\left(C_{k}, A\right) \geq c d(\Phi, \mathbf{Z})^{2} \sqrt{k}, \quad \text { with } \quad c=\frac{\pi^{2}}{2^{15} \sqrt{1+\frac{1}{k^{3}}}} \geq \frac{\pi^{2}}{2^{15} \sqrt{2}},
$$
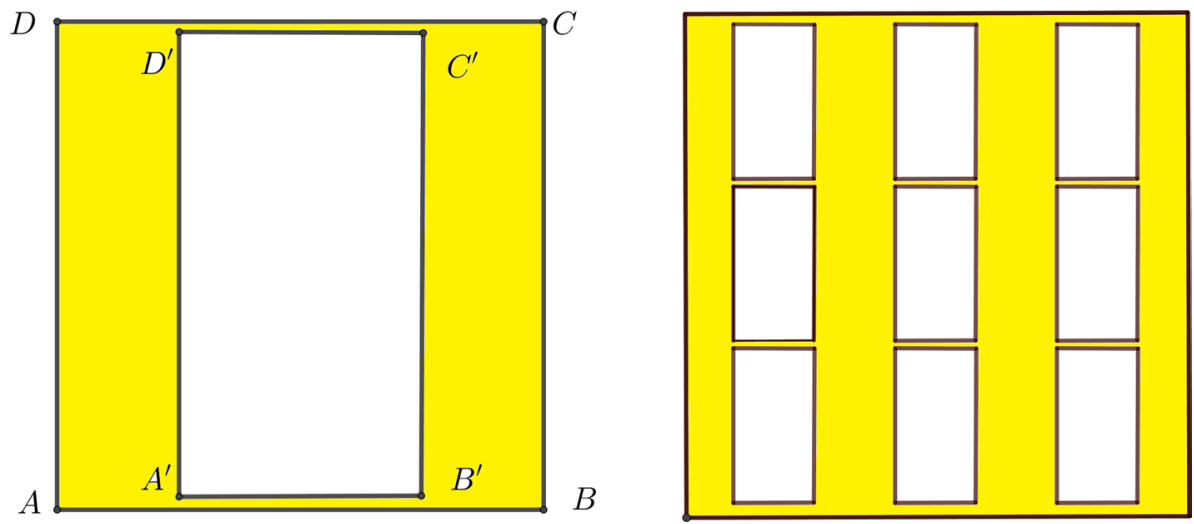

Fig. 2 On the left, the fundamental piece $C_{k}$ constructed with the rectangles $A B C D$ and $A^{\prime} B^{\prime} C^{\prime} D^{\prime}$. On the right, the domain $\Omega_{k}$ for $k=3$ obtained by assembling nine fundamental pieces together 
which grows like $\sqrt{k}$.

Step 2: the definition of $\Omega_{k}$ and its first eigenvalue. The domain $\Omega_{k}$ is a square of size 4 filled with $k^{2}$ copies of $C_{k}$ as in the picture. That is:

$$
\Omega_{k}=\cup_{j=1}^{k^{2}} C_{k j}
$$

where $C_{k j}$ is congruent to $C_{k}$ for all $j . \Omega_{k}$ has $k^{2}$ holes and $|\Omega|=k^{2}\left|C_{k}\right|=8+\frac{4}{k^{3 / 2}} \geq 1$.

We consider a potential $A$ having fixed flux $\Phi$ around each hole. First, it is easy to show that

$$
\lambda_{1}\left(\Omega_{k}, A\right) \geq \lambda_{1}\left(C_{k}, A\right) \geq c d(\Phi, \mathbf{Z})^{2} k^{\frac{1}{2}}
$$

where $c$ is the constant in Formula (21). In fact, let $u$ be a first eigenfunction of $\Omega_{k}$. By restricting it to each piece $C_{k j}$ we have, by the min-max principle:

$$
\int_{C_{k j}}\left|\nabla^{A} u\right|^{2} \geq \lambda_{1}\left(C_{k j}, A\right) \int_{C_{k j}}|u|^{2}=\lambda_{1}\left(C_{k}, A\right) \int_{C_{k j}}|u|^{2} .
$$

We sum over $j=1, \ldots, k^{2}$ and obtain

$$
\int_{\Omega_{k}}\left|\nabla^{A} u\right|^{2} \geq \lambda_{1}\left(C_{k}, A\right) \int_{\Omega_{k}}|u|^{2}
$$

which immediately gives $\lambda_{1}\left(\Omega_{k}, A\right) \geq \lambda_{1}\left(C_{k}, A\right)$.

Step 3: calculation of $h\left(\Omega_{k}\right)$. Now, let us see the total length $h$ of the segments we have to cut in order to make $\Omega_{k}$ simply connected. As the holes are at distance $\frac{2}{k^{5 / 2}}$, we need to cut $(k-1) k$ segments of length $\frac{2}{k^{5 / 2}}$ and $k$ segments of length $\frac{1}{k^{5 / 2}}$.

The total length we need to cut is $h\left(\Omega_{k}\right)=\frac{2 k-1}{k^{3 / 2}}$. Summarizing we have, as $k \rightarrow \infty$ :

$$
\left|\Omega_{k}\right| \geq 1, \quad h\left(\Omega_{k}\right) \sim \frac{2}{\sqrt{k}}, \quad \lambda_{1}\left(\Omega_{k}, A\right) \geq \operatorname{cd}(\Phi, \mathbf{Z})^{2} \sqrt{k},
$$

with $c \geq \frac{\pi^{2}}{2^{15} \sqrt{2}}$, which in particular shows that $\left|\Omega_{k}\right|$ is bounded from below, $h\left(\Omega_{k}\right)$ tends to zero and $\lambda_{1}\left(\Omega_{k}, A\right)$ tends to infinity, as requested.

Acknowledgements Research partially supported by INDAM and GNSAGA of Italy

The authors wish to thank the referee for his remarks, in particular, for the three references $[1,7,9]$ which are relevant not only for this paper but more generally for the problem of finding lower bounds for the ground state energy.

Funding Open access funding provided by Università degli Studi di Roma La Sapienza within the CRUICARE Agreement.

Open Access This article is licensed under a Creative Commons Attribution 4.0 International License, which permits use, sharing, adaptation, distribution and reproduction in any medium or format, as long as you give appropriate credit to the original author(s) and the source, provide a link to the Creative Commons licence, and indicate if changes were made. The images or other third party material in this article are included in the article's Creative Commons licence, unless indicated otherwise in a credit line to the material. If material is not included in the article's Creative Commons licence and your intended use is not permitted by statutory regulation or exceeds the permitted use, you will need to obtain permission directly from the copyright holder. To view a copy of this licence, visit http://creativecommons.org/licenses/by/4.0/. 


\section{References}

1. Balinsky, A.A.: Hardy type inequalities for Aharonov-Bohm magnetic potentials with multiple singularities. Math. Res. Lett. 10(2-3), 169-176 (2003)

2. Colbois, Bruno, Soufi, Ahmad El, Ilias, Said, Savo,Alessandro.: Eigenvalues upper bounds for the magnetic Schroedinger operator. to appear in Communication on Analysis and Geometry, arXiv:1709. 09482 (2021)

3. Colbois, Bruno, Maerten, Daniel: Eigenvalues estimate for the Neumann problem of a bounded domain. J. Geom. Anal. 18(4), 1022-1032 (2008)

4. Colbois, Bruno, Savo, Alessandro: Lower bounds for the first eigenvalue of the magnetic Laplacian. J. Funct. Anal. 274(10), 2818-2845 (2018)

5. Colbois, Bruno, Savo, Alessandro.: Lower bounds for the first eigenvalue of the Laplacian with zero magnetic field in planar domains, arXiv:2006.12762 (2020)

6. Fournais, S., Helffer, B.: Inequalities for the lowest magnetic Neumann eigenvalue. Lett. Math. Phys. 109(7), 1683-1700 (2019)

7. Frank, Rupert L., Hansson,Anders M.: Eigenvalue estimates for the Aharonov-Bohm operator in a domain. In Methods of spectral analysis in mathematical physics, volume 186 of Oper. Theory Adv. Appl., pages 115-137. Birkhäuser Verlag, Basel, (2009)

8. Helffer, B., Hoffmann-Ostenhof, M., Hoffmann-Ostenhof, T., Owen, M.P.: Nodal sets for groundstates of Schrödinger operators with zero magnetic field in non-simply connected domains. Comm. Math. Phys. 202, 629-649 (1999)

9. Laptev, Ari, Weidl,Timo: Hardy inequalities for magnetic Dirichlet forms. In: Mathematical results in quantum mechanics (Prague, 1998), volume 108 of Oper. Theory Adv. Appl., pages 299-305. Birkhäuser, Basel, (1999)

10. Shigekawa, I.: Eigenvalue problems for the Schrödinger operator with the magnetic field on a compact Riemannian manifold. J. Funct. Anal. 75, 92-127 (1987)

Publisher's Note Springer Nature remains neutral with regard to jurisdictional claims in published maps and institutional affiliations. 